from a developing country. Patients injured on the platform and off the platform had the same severity of injuries. This analysis shows the need for safety measures and strict law enforcement both at the station and at the track.

Prehosp Disaster Med 2019;34(Suppl. 1):s172-s173

doi:10.1017/S1049023X19003959

\section{Transformative Surgical Team Training}

Mrs. Amanda Gill, Mr. David Read, Ms Jodie Williams, Ms Annette Holian

National Critical Care \& Trauma Response Centre, Marrara, Australia

Introduction: Sudden onset disasters exceed the capabilities of local health services. Emergency Medical Teams (EMTs), including the Australian Medical Assistance Team (AUSMAT), are a vital element of the Australian Governments capacity to respond to regional and international sudden-onset disasters. AUSMAT has the capacity to deploy an EMT Type 2 surgical field hospital and has been successfully verified by the World Health Organisation (WHO). All AUSMAT members must complete AUSMAT Team Member training. The National Critical Care and Trauma Response Centre, Darwin, Australia is responsible for all AUSMAT training.

Aim: To educate and train the Surgical Team (perioperative nurses, surgeons, and anesthetists) in preparation for AUSMAT deployments in the austere environment.

Methods: Prior to 2015, the surgical AUSMAT training was conducted via two courses: one for perioperative nurses and a separate course for surgeons and anesthetists. In 2015, the course was redesigned with the aim of collaborative training with all the Surgical Team Members. The new Surgical Team Course (STC) engages all three professions to learn alongside each other and discuss potential difficulties in techniques, the daily running of the operating room, and ethical discussions.

Results: Since the rejuvenation of the STC, 15 surgeons, 17 anesthetists, and 18 perioperative nurses have completed the course. The attendees are familiarized with operational and clinical guidelines, the surgical field hospital, and operating room equipment including CSSD. A pivotal component of the course focuses on the essentials of medical records and Minimum Data Set reporting for EMTs as defined by WHO. Discussion: Since 2015, the NCCTRC has successfully run two courses. The revised collaborative model for AUSMAT STC has enhanced the quality of the program and subsequent learning experiences for participants.

Prehosp Disaster Med 2019;34(Suppl. 1):s173

doi:10.1017/S1049023X19003960

Triage Problem Among the Ambulance Crew (Paramedic) in Japan

Dr. Keiji Nakata

Social Studies of Disaster Management, Kobe Gakuin University, Kobe, Japan

Introduction: At various accidents or disaster sites, rescue, first aid, and transport to hospitals has been provided by ambulance crews (paramedics). In the case of mass casualties, they also need to operate triage for injured people.

Aim: To consider and reveal challenges in triage by ambulance crews (paramedics) on-site.

Methods: Interviews of seven ambulance crews (paramedics) and their instructors were conducted and their answers were analyzed.

Results: (1.) Triage black tags: declaring "deceased: not able to survive" might give a heavy mental burden and psychological responsibility. Legal protection and an interstitial rule will be necessary in the future. (2.) Missed triage: the ambulance crew cannot perform a triage that may develop a legal problem. It is always important to prevent ambulance crews from being charged. (3.) Triage education and training: there are few triage trainings at fire departments although the number of emergency medical responses is increasing compared to fire response. It will be necessary to increase time of the triage education and training in near future. (4.) Command system (characteristic rank system in the fire department): There is a problem with the rank system in fire departments since confusion occurs when a commander of the First Aid Station is not a licensed paramedic. The ambulance crew (paramedic) usually consists of the three different ranked people. Individual operations are difficult during operation. Education for the paramedic executive is necessary for the fire organization.

Discussion: For the triage by ambulance crew (paramedic), legal protection by medical control operation is required, and it may lead to a reduction of heavy mental burden. Triage training is needed to improve the training of triage. The ambulance crew (paramedic) operates under the fire department command system. However, at the time of disaster, the ambulance crew (paramedic) should also work under the medical command system.

Prehosp Disaster Med 2019;34(Suppl. 1):s173

doi:10.1017/S1049023X19003972

Unexpected Lessons from a Mass Casualty Simulation: Strategies for Management of the Minimally Injured Can Increase Efficiency and Decrease Chaos

Dr. Joseph McIsaac ${ }^{1}$, Dr. Brenda Gentz ${ }^{2}$, Dr. Patricia McFadden ${ }^{1}$, Dr. John Coleman ${ }^{3}$

1. University of Connecticut School of Medicine, Farmington, United States

2. University of Arizona, Tucson, United States

3. Virginia Mason Medical Center, Seattle, United States

Introduction: The SALT Triage system has been advocated as an easy-to-use sorting and treatment system for mass casualty incidents (MCI). Minimally injured (GREEN) patients tend to be in the majority and may cause impediments to access and treatment of the most critically injured (RED). By identifying flaws in MCI communications that impair effective patient care, responders can be more effective.

Aim: To discover strategies that effectively manage the minimally injured and leverage their help, increasing triage efficiency and treatment of the immediate casualties.

Methods: Direct observation, after-action debriefing, and literature search. 
Results: The literature was vague regarding recommendations on a bystander and trained provider communication best practices. Feedback from standardized patients (actors) and participants during a structured debriefing following a 2018 American Society of Anesthesiology MCI exercise suggested that triaging providers under stress may communicate poorly, contributing to increased patient anxiety, disruptive behavior, and less effective team dynamics during a disaster. Strategies suggested include: eye contact; therapeutic touch (culturally appropriate); using slow, clear, reassuring speech; clearly explaining what is happening and why (sickest (RED and YELLOW) first priority, minimal (GREEN) next, expectant (BLACK) last); acknowledging their emotional state and their grief (not ignoring them); assigning nontechnical tasks to those capable of helping (putting pressure on a wound, moving casualties, or comforting the injured, dying, and the emotionally distraught).

Discussion: Bystander engagement has been repeatedly identified as a means to increase the capacity of first responders to provide care to patients during an MCI. Utilization and management of the minimally injured and any uninjured bystanders and responders can become a force multiplier for the triage/ treating responders. Developing a best practice dialogue to be used in training first responders could help improve many of these issues and augment current MCI training programs.

Prehosp Disaster Med 2019;34(Suppl. 1):s173-s174

doi:10.1017/S1049023X19003984

\section{Uniform Guideline on Risk Characterization for Approval} of Mass Gatherings

Vincent Pot Msc ${ }^{1}$, Johan de Cock PhD' ${ }^{2}$, Jan van Leeurwen ${ }^{3}$

1. GGD GHOR Nederland, Utrecht, Netherlands

2. Anaphem, Nijmegen, Netherlands

3. Safety Region Rotterdam Rijnmond, Rotterdam, Netherlands

Introduction: Mass gatherings may have far-reaching effects on medical care because of the potential high load on the health care system. In preparation of large events (mass gatherings), such as music festivals or marathons, an extended risk assessment forms the basis for issuing advice on health and safety and possible necessary precautionary measures. In the Netherlands, the 25 regional Public Health Directors are responsible for public health and safety. This includes responsibility for advice on large-scale events, based on which the local authorities (e.g. the mayor) decide on the approval. Health care professionals are looking for better tools with regard to the arising dilemmas around responsibilities and risks. Also, as new forms of events are created, uniform (safety) regulations are lacking. GGD GHOR Netherlands (Dutch Society of the regional Public Health Services [GGD] and Major Incident Medical Planning and Coordination Offices [GHOR]) has updated the existing national guideline in collaboration with the Academic Network for Applied Public Health and Emergency Management (Anaphem). The focus was on improving the guideline by including all current expertise and experience in the field. Methods: Various expert sessions were held in 2017 and 2018 to collect all relevant knowledge, evidence, and experience that is currently accessible to develop an improved uniform approach for risk assessment and process steps.

Results: A new dynamic national guideline, including factsheets in various topics being effective by 2019 . As a result, a list of topics is formulated for further research.

Discussion: The new guideline includes the current knowledge and raised awareness among the experts of some important gaps in current evidence on several topics.

Prehosp Disaster Med 2019;34(Suppl. 1):s174

doi:10.1017/S1049023X19003996

The University of Colorado Graduate Medical Education Fellowship in Climate Change and Health Science Policy Dr. Chelsea Dymond ${ }^{1}$, Dr. Cecilia Sorensen ${ }^{2}$, Dr. Emilie Calvello-Hynes', Dr. Jay Lemery ${ }^{2}$

1. Denver Health Medical Center, Denver, United States

2. University of Colorado School of Medicine, Aurora, United States

Introduction: Climate change is intricately related to human health and impacts acute and chronic diseases leading to increased demands on the health care system.

Aim: The University of Colorado Graduate Medical Education (GME) Fellowship in Climate Change and Health Science Policy (CCHSP) aims to train and equip a new generation of clinicians knowledgeable in climate science, proficient in climate health education, and facile with advocacy skills in order to become leaders in health policy.

The CCHSP fellowship is funded by the Living Closer Foundation and hosted through the University of Colorado Department of Emergency Medicine. It is a one to two-year program tailored to the fellow's specific goals with the opportunity to earn an MPH or MA. Clinical work is supported through the UCHealth network. Site placement occurs at partnering organizations, including the National Institutes of Health, the Centers for Disease Control and Prevention, and fieldwork throughout the world (via Colorado School of Public Health, Harvard FXB Center for Health and Human Rights).

The first fellow was recruited in 2017 and has participated in and completed multiple projects: technical contributor to the US Government's Fourth National Climate Assessment; advocating for women's health policy in India; authorship of climate change and health resource documents for the World Bank; climate change leadership within SAEM; advocacy work with local and state governments; multiple research publications.

Discussion: As climate change continues to impact human health with widespread consequences, we need effective and articulate leaders to affect policy. Although this Fellowship originated in Emergency Medicine, its competencies and structure are replicable for other clinical specialties. Climate change will be one of the core global health challenges for generations. A strong foundation of clinicians who understand its causes and the strategies for adaptation and mitigations are necessary to optimize health outcomes amidst this growing threat.

Prehosp Disaster Med 2019;34(Suppl. 1):s174

doi:10.1017/S1049023X1900400X 